\title{
REPÉRAGES DISCURSIFS ET TRADUCTION
}

\begin{abstract}
Guillemin-Flescher Jacqueline, Repérages discursifs et traduction [Discoursive points of reference and translation]. Studia Romanica Posnaniensia, Adam Mickiewicz University Press, Poznań, vol. XXV/XXVI: 2000, pp. 145-155, ISBN 83-232-0965-0, ISSN 0137-2475.

In the article the problem of differences between texts in French and English is discussed in relative clauses, indicative (deictic) expressions and dislocations. It appears that no grammatical restrictions determine the use of one or another form in both languages discussed here. These are restrictions which should be considered on another level, namely, on the level of various ways of placing points of reference in French as related to the sender of the communication, and in English as related to its receiver.
\end{abstract}

\section{INTRODUCTION}

Lorsqu'on observe un corpus de textes traduits, avec mise en regard de l'original et de la traduction, il apparaît clairement qu'il existe des constantes systématisables dans le passage d'une langue à l'autre. Ces constantes ne constituent pas des phénomènes isolés; elles relèvent d'une explication théorique globale et indiquent qu'il existe, au delà de la grammaire de la phrase, des schémas discursifs qui sont particuliers aux langues, ou aux groupes de langues. Les contraintes discursives sont, bien entendu, transgressées dans une certaine mesure pour rendre compte d'effets stylistiques particuliers. Il n'en reste pas moins que dans de nombreux cas ces contraintes sont si fortes qu'une transgression apparaîtra comme a-grammaticale. Dès lors qu'il y a mise en discours, on s'aperçoit que le fonctionnement du langage ne dépend pas uniquement d'un agencement syntaxique autonome mais qu'il est largement conditionné par des choix énonciatifs qui sont la manifestation linguistique de différences culturelles. Ces différences apparaissent quels que soient les phénomènes linguistiques mis en jeu. Elles se vérifient aussi bien dans le système de détermination que dans l'agencement des procès, et la relation inter-énoncés, tous paramètres confondus. 
Je prendrai simplement à titre d'exemples deux langues: le français et l'anglais et trois phénomènes où se manifestent ces différences: les propositions relatives, la deixis et les phrases clivées et disloquées. On constate, dans chacun des cas, qu'en anglais une distinction radicale s'opère entre les relations qui posent l'existence d'un élément ou d'une occurrence événementielle et les énoncés qui qualifient un élément ou une relation dont l'existence a été explicitement posée.

\section{LES PROPOSITIONS RELATIVES}

Ainsi, lorsqu'on trouve en français une proposition relative qui exprime une situation délimitée dans le temps comme dans l'énoncé suivant:

«Mme X ...... regagna sa chambre qu'elle considéra un instant d'un air agacé»

on constate que, de façon quasi systématique, les traducteurs la rendent par une proposition indépendante en anglais. J'ai testé quinze anglophones, en leur demandant de traduire cet énoncé. Il était inséré dans son contexte de façon à ce qu'ils ne sachent pas ce que je testais. Tous ont traduit «qu'elle considéra un instant d'un air agacé» par une proposition coordonnée. J'ai demandé ensuite à mes informateurs s'ils auraient accepté une proposition relative. Ils ont tous répondu «non» de façon catégorique. Les linguistes ont même été bouleversés par leur propre réaction. Ils pensaient a priori qu'une proposition relative devait nécessairement pouvoir se traduire par une proposition relative dans d'autres langues. Aucune contrainte grammaticale répertoriée par les grammaires ne l'interdit. Or, de toute évidence une traduction littérale était ressentie par les anglophones comme a-grammaticale. Un relevé fait sur 15 ouvrages comportant des types de discours diversifiés a fait apparaître des critères définissables qui déterminent la traduction d'une relative, en français, par une proposition indépendante en anglais. Comme en témoignent les exemples [1] à [4] le degré de contrainte sera plus fort

- si la relative porte sur le complément d'objet de la principale,

- si celui-ci est faiblement déterminé, par exemple lexicalement',

- si le sujet de la relative a un statut agentif,

- si le procès construit un instant dans la narration.

Les deux derniers critères peuvent jouer conjointement ou isolément

[1].. je bute vers un second battant que je pousse cette fois vers l'intérieur.

I came to a second door and this time pushed it inward.

(J. Malaurie, Les derniers rois de Thulé, p. 147/97)

[2] Les hommes dévorent donc une viande rêche et bouillie que les délicats détachent avec leurs ongles.

${ }^{1}$ Par détermination, j'entends non seulement la détermination nominale mais tout ce qui fait partie intégrante de la construction linguistique d'un objet, y compris les propriétés lexicales associables au nom. Le terme détermination s'oppose en ceci à la qualification qui suppose un objet déjà construit. 
The men chomped ... on this tough boiled meat. The more delicately mannered used their fingernails.

(J. Malaurie, Les derniers rois de Thulé, p. 52/27)

[3]... Boissier se dirigea vers une des fenètres, sur laquelle il laissa ... traîner ses doigts, puis il adressa un signe d'intelligence à Maigret.

... Boissier crossed to one of the windows, ran his fingers over it, then gave Maigret a signifant nod.

(G. Simenon, Maigret et la grande perche)

[4] Puis il fit un brusque demi-tour et s'enfuit ventre à terre dans le taillis où il disparut.

He turned abruptly and bolted into the wood.

(M. Tournier, Vendredi ou les limbes du Pacifique, p. 32/30)

Dans chacun de ces exemples, en français on qualifie par une proposition relative, un élément de la proposition principale qui est faiblement déterminé

- d'une part en raison de sa position syntaxique (complément d'objet) qui implique un statut de dépendance,

- d'autre part en raison de ses propriétés sémantiques (terme désignant un inanimé battant/viande /fenètres/taillis).

En anglais, dans chacun des cas l'énonciateur transforme la relative en proposition indépendante ou coordonnée et construit une nouvelle relation au lieu de qualifier la précédente.

Ces contraintes ne peuvent pas être considérées comme réversibles. Toutefois les corpus de textes traduits de l'anglais vers le français font apparaître une proportion élevée de schémas différentiels du même type:

[5] Denis ...shouted 'hooray', and was quite properly reproved by one of the sergeants at arms. Denis .. poussa un hourra qui lui attira, à juste titre, les réprimandes du sergent d'armes.

(M. Thatcher. The Downing Street Years, p. 3/13)

[6] Khamel fired three bullets into the back of his head, and he fell loudly on the kitchen table. Khamel tira trois balles dans la nuque du policier qui s'effondra lourdement sur la table de la cuisine.

(G. Grisham, The Pelican Brief, p. 27/38)

Dès lors que la contrainte concernant la construction d'existence n'est plus pertinente, la différence entre les deux langues disparaît: autrement dit, les relatives pourront apparaître en anglais comme en français. Les conditions qui le permettent demandent cependant à être précisées. Elles peuvent en effet être de plusieurs ordres:

- soit on qualifie un élément ou une situation déjà construite

[7]... Maigret franchit ... le portail flanqué de deux agents en uniforme qui se tenaient tout contre le mur.

... Maigret passed ... through the gate flanked by two uniformed policemen who were standing right up against the wall.

(G. Simenon, La colère de Maigret, p. 7)

- soit on prédique une propriété ou un constat de fait

[8] The place is full of people who hate Coal.

Il y a là bas des tas de gens qui ne peuvent pas voir Coal en peinture.

(G. Grisham, The Pelican Brief, p. 86/103) 
- soit encore on qualifie un élément par une relation non validée (c'est à dire une relation qui est envisagée mais non réalisée telle qu'une hypothèse ou une visée)

[9] Inflation was a monetary phenomenon which it would require monetary discipline to curb. L'inflation était un phénomène monétaire qui ne pouvait être jugulé que par la discipline monétaire.

(M. Thatcher, The Downing Street Years, p. 33/39)

En [7] l'imparfait en français: qui se tenaient et la forme auxiliée en -ing en anglais: were standing impliquent un repérage par rapport à un élément déjà construit dans la principale: c'est à dire deux agents en uniforme.

En [8] le prédicat subjectif hate/voir en peinture exprime une attitude. L'agentivité n'est pas en jeu.

En [9] l'auxiliaire modal would/pouvait indique que la réalisation du procès est hypothétique. Aucun de ces énoncés n'atteint le degré de détermination qui exige une proposition indépendante en anglais.

Ainsi en français, on peut qualifier une situation quelque soit son degré de détermination sans que son existence ait été construite dans le contexte antérieur, alors qu'en anglais, seules

- les relations et les éléments dont l'existence a déjà été explicitement posée, et

- les prédications dont le procès est faiblement déterminé, sont compatibles avec une qualification de type relative non déterminative ${ }^{2}$. Comment cette différence peut-elle s'expliquer? Mon hypothèse est la suivante: dans la mise en place des déterminations, en français le point de vue est celui de l'énonciateur, ce qui permet de pré-construire une première relation sans avoir à la poser explicitement. En anglais, les déterminations sont calculées par rapport au co-énonciateur, ce qui a pour conséquence

1) le fait de poser explicitement toutes les opérations, de détermination et

2) d'envisager aussi bien les relations intra qu'inter-énoncés dans un repérage contextuel.

${ }^{2}$ Faute d'espace, le problème des relatives non déterminatives ne peut pas être traité ici. Sans prétendre que la distinction entre deux types de relatives soit systématiquement pertinente, il convient de signaler que dans les cas non-ambigus, les déterminatives donnent également lieu sous certaines conditions à des transformations systématisables en anglais: à titre d'exemple, la traduction par un syntagme prépositionnel: il commença à rassembler les provisions qu'il embraquerait avec lui / He started to collect provisions for the journey (M. Tournier, Vendredi ou les limbes du Pacifique, p. 35 / N. Dennis. p. 32); Je suis un misérable ... j'ai bu le rhum qui se trouvait dans le coffre /I'm a miserable wretch... I drank the rum from the locker (Hergé, Tintin, Le crabe aux pinces d'or, p. 20 / L. Lonsdale-Cooper, M. Turner, p. 20). Dans le sens anglais-français: She had shattered the communion between them / Elle avait brisé la communion qui s'était établie entre eux (F. O'Connor, The Complete Stories, p. 439 / M. Gresset, C. Richard, p. 151). Alors que dans le cas des non-déterminatives on construit une nouvelle relation, dans le cas des déterminatives on intègre la localisation à un terme existant de façon à ce qu'elle participe à sa construction. Dans un cas comme dans l'autre on passe de la qualification à la construction d'un objet ou d'une relation. 
En français, le repérage dominant se situera au contraire entre le point origine de l'énonciation et les éléments ou situations mises en place.

Cette différence se vérifie dans des phénomènes qui pourraient sembler a priori n'avoir aucun rapport avec les contraintes qui régissent la distinction subordonnée/principale. Ainsi, la deixis, tout en relevant comme les relatives de la linguistique générale, n'a cependant pas un fonctionnement identique d'une langue à l'autre.

\section{LA DEIXIS}

Prenons d'abord le cas où l'on désigne plusieurs éléments qui relèvent

- soit d'un domaine notionnel commun,

- soit d'une situation commune.

On constate que sous ces conditions,

- en français, chaque terme sera précédé d'un déterminant déictique,

- en anglais le premier terme seulement; dès lors que ce premier repérage est mis en place, c'est la relation contextuelle entre les termes désignés qui sera de rigueur.

[10] Fernand Point, qui reste mon maître, a supprimé dès avant la guerre toutes ces sauces, ces plats compliqués, trop riches, ces garnitures qui faisaient loi dans la cuisine de $\mathrm{XIX}^{\mathrm{e}}$ siècle. Before the last war Fernand Point, whom I regard as my master, had already dispensed with all the sauces, complicated dishes and elaborate garnishes which were the rule during the nineteenth century.

(P. Bocuse, La cuisine du marché, p. 6/xv)

[11] Ils venaient pourtant de loin, ces hommes et ces femmes de tous âges qui se pressaient à nos cours avec une ferveur soupçonneuse...

Yet these men and $\emptyset$ women of all ages who crowded into our lecture-rooms with a mixture of enthusiasm and suspiciousness had a lot of leeway to make up.

(C. Lévi-Strauss, Tristes Tropiques, p. 114/101)

[12] Mais ces rapprochements et ces distinctions ne surprennent pas le sentiment esthétique... These connections and $ø$ distinctions are however no surprise to our aesthetic sense.

(C. Lévi-Strauss, La pensée sauvage, p. 20/12)

[13] ... ces cimes glacées, ces grottes et ces forêts profondes [...] ce sont, à des titres divers, les ennemis d'une société qui se joue elle-même la comédie...

...these icy summits, $\emptyset$ deep caverns and $\emptyset$ impenetrable forests [...] are all, in their different ways, enemies of our society, which pretends to itself...

(C. Lévi-Strauss, Triste Tropiques, p. 42/31)

Lorsque chaque terme est désigné par deixis, c'est la relation entre l'énonciateur et l'élément désigné qui est privilégié; la propriété commune qui les constitue en classe n'est pas envisagée. En anglais, en revanche, ils sont envisagés en tant qu'ensemble d'éléments appartenant à une même situation ou un même domaine notionnel. 


\section{OCCURRENCES SPÉCIFIQUES EN FRANÇAIS $\rightarrow$ OCCURRENCES HYPOTHÉTIQUES OU QUELCONQUES EN ANGLAIS}

Ceci ne vaut cependant que pour les termes dont l'existence a été posée. Dès lors que leur existence est hypothétique, en anglais, il y a incompatibilité avec un repérage déictique. En français, ce critère n'a aucune incidence sur la nature du repérage. Prenons un exemple: au cours d'un match de hockey sur glace aux jeux olympiques, un commentateur a prononcé les paroles suivantes:

[14] J'espère vous annoncer bientôt cette victoire et ce soir cette qualification.

Il s'agit de la victoire hypothétique d'une équipe française en vue d'une qualification non moins hypothétique dans un tournoi. La victoire n'étant pas acquise au moment de l'énonciation, seul le génitif peut établir en anglais la relation exprimée en français par la désignation déictique: cette victoire / cette qualification (their victory, their qualification)

Dans l'exemple (15)

[15]... nulle forme d'art ne mériterait ce nom si elle se laissait capter tout entière par les contingences extrinsèques, que ce soit celle de l'occasion ou celle de la destination...

No form of art is [...] worthy of the name if it allows itself to come entirely under the sway of extraneous contingencies, whether of occasion or purpose.

(C. Lévi-Strauss, La pensée sauvage, p. 42/29)

le marqueur si et le subjonctif que ce soit indiquent clairement le caractère hypothétique du terme contingences extrinsèques. On constate cependant que ce terme est repris deux fois par un pronom démonstratif: celle de l'occasion ou celle de la destination alors qu'en anglais le repérage déictique disparait complètement: whether of $\phi$ occasion or purpose.

Voyons maintenant les exemples (16) et (17):

[16] Les moindres bruits parlent. Un son mat? C'est un rocher qui se détache de la falaise [...] un écho feutré et sourd? C'est un pan de neige qui glisse; ce névé qui brille? A contourner si possible, la neige y est molle. Cette glace noire? A éviter, elle se déroberait sous le pied. Cette glace blanche? Bonne, épaisse.

The smallest noises were eloquent. A dull thud? A rock had broken loose and fallen from the cliff [...] A low, muffled echo? A section of snow had slid down some slope. A patch of shining névé? Better skirt it, because there the snow was soft. Black ice? Avoid that, too, for it would give way under me. White ice? Good, thick...

(J. Malaurie, Les derniers rois de Thulé, p. 407/290)

[17]... le prix des produits locaux est incroyablement bas: cet ananas me coûterait vingt sous, ce régime de bananes deux francs, ces poulets qu'un boutiquier italien fait rôtir à la broche, quatre francs.

...the prices of local produce were incredibly low. A pineapple cost twenty sous, a bunch of bananas two francs, $\emptyset$ chickens, spit-roasted by an Italian shopkeeper, four francs each.

(C. Lévi-Strauss, Tristes tropiques, p. 95/81-82)

Ces exemples ont en commun avec (14) et (15) le fait que $C$ 'est un rocher/C'est un pan de glace, etc. cet ananas/ce régime de bananes etc. désignent des termes dont 
l'occurrence n'est pas validée. En revanche la non-validation n'est pas du même ordre que dans le cas des hypothétiques. Il s'agit cette fois d'éléments quelconques dans une série de mises en situations simulées, qui renvoient simplement à une occurrence dans une classe de possibles.

Les démonstratifs sont à nouveau traduits en anglais par d'autres déterminants: article indéfini a, au singulier, article $\emptyset$ au pluriel.

D'un point de vue théorique les deux cas de figure: occurrence hypothétique et occurrence quelconque obéissent à une même contrainte, du fait que leur existence n'est construite ni dans l'un ni dans l'autre cas.

\section{DEIXIS ET RELATION INTER-ÉNONCÉS}

Examinons à présent la deixis lorsqu'elle intervient non plus entre plusieurs éléments d'une même classe, mais dans la relation inter-énoncés. On constatera que la contrainte concernant le contexte antérieur est du même ordre que pour les relatives.

En français, la première occurrence du terme qui est repris ensuite par deixis peut être préconstruite, c'est à dire posée sans être explicitée et ceci quelle que soit la situation énonciative; en anglais, la préconstruction ne sera possible qu'en situation. La deixis situationnelle se différencie en effet de la deixis contextuelle en ceci qu'il y a co-présence des deux énonciateurs. Dans une relation discursive, en revanche, l'identification d'un terme qui n'a pas été explicitement posé ne peut se faire qu'à partir d'un point de référence: celui de l'énonciateur.

\section{POINT DE RÉFÉRENCE ET DÉTERMINATION}

Qu'il s'agisse d'énoncés traduits ou d'énoncés produits dans la langue d'origine, cette différence entre l'anglais et le français semble fondamentale. Elle indique des fonctionnements métalinguistiques radicalement différents. En français, il suffit que l'identification du terme désigné se fasse par rapport à l'énonciateur. En anglais, l'identification doit se faire par rapport au double point de référence: énonciateur et co-énonciateur. Cela explique pourquoi dans les exemples (18) à (20) un pronom déictique apparait dans les deux langues.

[18] L'utilisation des ressources naturelles dont disposaient les indigènes hawaiiens était, à peu de choses près, complète ; bien plus que celle pratiquée dans l'ère commerciale actuelle...

These native Hawaiians' utilization of their available natural assets was well-nigh complete - infinitely more so than that of the present commercial era...

(C. Lévi-Strauss, La pensée sauvage, p. 6/3)

[19] Car (...) l'effort du portraitiste (...) pour capter sur sa toile l'expression la plus révélatrice (...) de son modèle, fait partie du mème genre que celui d'un Detaille...

For (...) the attempt of a portrait painter (...) to recapture on his canvas his model's most revealing expression (...) belongs to the same genre as that of a painter like Detaille...

(Ibid., p. 41/28) 
[20] La structure formelle de ce qui, au premier abord, pourraît apparaître comme une compétition sportive, est en tous points similaire à celle d'un pur rituel...

The formal structure of what might at first sight be taken for a competitive game is in fact identical with that of a typical ritual...

(Ibid., p. 46/32)

Dans chacun de ces énoncés on compare le terme désigné par un déictique avec un terme antérieur, c'est à dire le terme indiqué en gras. Dans les exemples (21) et (22) en revanche, aucun contexte antérieur n'autorise l'emploi d'un adjectif démonstratif en anglais. Comme en témoignent les énoncés d'origine, en français ce critère n'est pas contraignant. Les déictiques apparaissent indifféremment dans les deux séries d'exemples.

[21] Un petit portrait d'elle que j'ai gardé me la montre telle qu'elle était alors [...] dans cette pose qui lui était si coutumière...

I still have by me a little portrait of her, in which I can see her as she then was [...] sitting sideways in an attitude which was habitual to her...

(A. Gide, La porte étroite, p. 16/12)

[22] On s'est longtemps plu à citer ces langues où les termes manquent, pour exprimer des concepts tels que ceux d'arbre ou d'animal...

It has long been the fashion to invoke $\emptyset$ languages which lack the terms for expressing such a concept as 'tree' or 'animal'...

(C. Lévi-Strauss, La pensée sauvage, p. 3/1)

\section{STRUCTURES DISLOQUÉES ET STRUCTURES CLIVÉES}

Les structures clivées et disloquées, à savoir les constructions du type «Ils sont fous ces romains» et «Ce n'est pas l'homme qui partage vos envies qui vous dira le contraire» posent des problèmes complexes de traduction. Je n'évoquerai ici que les points concernant la nature des repérages dans les deux langues envisagées.

On constate tout d'abord que la majorité de ces structures se traduisent en anglais par un énoncé canonique de type sujet verbe objet. En ce qui concerne les structures disloquées, le dédoublement d'un même terme, par exemple par un nom et un pronom anaphorique, n'est possible en anglais que si l'un et l'autre sont pris dans des relations distinctes. En français en revanche les deux termes peuvent être pris dans une même relation

[23] Il ne pouvait pas descendre pour nous la poser, son énigme, votre bonhomme?

Couldn't this old man come down here to ask us his riddle?

(Les 12 travaux d'Astérix, p. 42)

[24] De le voir ainsi cet ignoble cavalier dans une tenue aussi peu réglementaire, et tout foirant d'émotion, ça le courrouçait fort notre colonel.

It made our colonel very angry to see that wretched cavalryman so incorrectly dressed and shitting in his pants with fright.

(Céline, Voyage au bout de la nuit, p. 16/21) 
[25] We had finally been arguing that case for the best part of four years...

Ce dossier, nous l'avons en fait soutenu pendant près de quatre années.

(M. Thatcher, The Downing Street Years, p. 2/15)

[26] They discovered that she couldn't have children so they found me.

Quand ils ont découvert qu'elle était stérile, ils m'ont trouvée, moi.

(PD James, Innocent Blood, p. 10/17)

En français, deux termes co-référentiels apparaissent dans un même énoncé (Il/votre bonhomme; la/son énigme). Le terme détaché est soit repris soit redésigné dans la relation prédicative. En anglais, sauf cas marginaux, on ne pourra avoir co-référence entre deux termes dans un même énoncé que si l'identification est explicitée par une copule, exemple: London is the capital of England. Elle devra sinon être établie par repérage avec un premier terme dont l'existence est posée dans une prédication antérieure ou en situation.

Le repérage par rapport à un terme antérieur vaut de la même façon pour les phrases clivées. Celles-ci n'apparaîtront en effet en anglais que lorsqu'une relation, soit de reprise, soit de contraste, établit une relation contextuelle entre un terme dont l'existence est posée dans une première relation et le terme thématisé dans la structure clivée.

[27] Ça a débuté comme ça. Moi, j'avais jamais rien dit. Rien. C'est Arthur Ganate qui m'a fait parler.

Here's how it started. I'd never said a word. It was Arthur Ganate that made me speak up.

(Celine, Voyage au bout de la nuit, p. 7/13

[28] When you were adopted you were given a new birth certificate, and the information which links your present name, Philippa Rose Palfrey, with your original birth certificate is kept by the Registrar General in confidential records. It is this linking information which the law now requires the Registrar General to give you if you want it.

Lors de votre adoption, vous avez reçu un nouvel extrait de naissance. Le renseignement qui relie votre nom actuel, Philippa Rose Palfrey, à votre extrait de naissance originel, se trouve dans les dossiers confidentiels de l'état civil. C'est ce renseignement-chaînon que la loi permet de donner à présent.

(P.D. James, Innocent Blood, p. 6/11)

[29] ...thursday was a day of more than ceremonial importance....... It was on that day that Helmut Schmidt, the West German Federal Chancellor, arrived in London on an official visit...

... le jeudi avait une importance qui allait au-delà des questions de cérémonie... C'était ce jour là que Helmut Schmidt, chancelier de RFA, venait à Londres pour une visite officielle..

(M. Thatcher, The Downing Street Years, p. 34/41)

Le fait qu'en français le repérage contextuel avec une relation antérieure n'est pas une contrainte explique la disparité dans la fréquence de cette structure dans les deux langues. 


\section{CONCLUSION}

Les différences que je viens de souligner entre l'anglais et le français sont liées, comme je l'ai indiqué au départ, à des types de repérage différents selon les langues. Les variations se manifestent au niveau de la syntaxe, mais celle-ci n'est que la trace d'opérations qui se situent à un autre niveau. L'explication des différences observées me semble résider en ceci, qu'en français on tend de façon prédominante à construire les valeurs référentielles par rapport à l'énonciateur, en anglais par rapport au co-énonciateur. Ces repérages ont des conséquences fondamentales à la fois sur le plan théorique et dans l'opération de traduction. En effet, si le point de référence est l'énonciateur, la première opération pourra aisément être préconstruite. En revanche, si le point de référence est le co-énonciateur, la construction de même que la reconstruction des valeurs référentielles ne pourra se faire qu'à partir d'opérations explicitées dans l'énoncé ou définies situationnellement par rapport à un double point de référence.

\section{BIBLIOGRAPHIE}

\section{OUVRAGES ET ARTICLES THÉORIQUES}

Culioli A. (1990), Pour une linguistique de l'énonciation, tome 1, Paris, Ophrys.

Fuchs C., Milner J. (1979), A propos des relatives, Paris, SELAF.

Guillemin-Flescher J. (1981), Syntaxe comparée du français et de l'anglais: problèmes de traduction, Paris, éditions Ophrys.

- (1993), Etude contrastive de la deixis en anglais et en français, in: Opérations énonciatives et interprétation de l'énoncé, Paris, Ophrys, pp. 181-208.

- (1998), Qualification and point of view, in: Function and Structure: Festschrift for Susumu Kuno, Akio Kamio et Ken-ichi Takami (eds.), John Benjamins, Amsterdam and Philadelphia, (sous presse).

Kleiber G. (1992), Anaphore, deixis: deux approches occurrentes, in: La deixis, Paris, P.U.F., pp. 613-626.

Kuroda S.Y. (1968), English Relativization and certain related Problems, Language, vol. 4, $\mathrm{N}^{\circ} 2$, pp. 214-266.

Muller C. (1996), La subordination en français, Paris, Armand Colin.

CORPUS

Bocuse P. (1976), La cuisine du marché, Paris, Flammarion.

Traduction anglaise: Rossant C., Davis L., Londres-Toronto-Sydney-New York, Granada Publishing Ltd, Frogmore, St Albans, Herts, 1978.

Céline L.F. (1952), Voyage au bout de la nuit, Paris, Gallimard, «Folio».

Traduction anglaise: Manheim R., Londres, John Calder Ltd., 1988. 
Gide A. (1976), La porte étroite, Paris, Mercure de France, 1909, Gallimard, «Folio».

Traduction anglaise: Bussy D., Londres, Secker et Warburg, 1924; Harmondsworth, Middlesex, Penguin Modern Classics, 1952.

Goscinny R., Uderzo A. (1976), Les 12 travaux d'Astérix, Neuilly sur Seine, Dargaud.

Traduction anglaise: (anonyme), Londres, Hodder \& Stoughton, 1978.

Grisham G. (1993), The Pelican Brief, Londres, Arrow Books Ltd.

Traduction française: Berthon P., Paris, Robert Laffont, «Pocket», 1993.

Hergé (1947, 1960), Le crabe aux pinces d'or, «Les aventures de Tintin», Paris, Tournai, Casterman, 1947, 1960

Traduction anglaise: Lonsdale-Cooper L., Turner M., Londres, Methuen \& Co Ltd, 1958; Magnet Edition, 1972, 1976.

James P.D. (1980, 1989), Innocent Blood, Penguin Books, Harmondsworth, Middlesex.

Traduction française: Rosenbaum L., Paris, Fayard Mazarine, 1984.

Lévi-Strauss C. (1955), Tristes Tropiques, Paris, Plon.

Traduction anglaise: Weightman J. \& D., Tristes Tropiques, Londres, Jonathan Cape Ltd, 1973, Atheneum, 1974; New York, Pocket Book, 1977.

- (1962), La pensée sauvage, Paris, Plon.

Traduction anglaise: (anonyme), Londres, Weidenfeld \& Nicolson, 1966, 1981.

Malaurie J. (1976), Les derniers rois de Thulé, Paris: Plon, collection «Terre Humaine».

Traduction anglaise: Foulke A., Londres: Jonathan Cape Ltd., 1982.

O'Connor F. (1946, 1971), The Complete Stories, New York, Farrar, Strauss et Giroux.

Traduction française: Gresset M., Richard C., Paris, Gallimard, 1975.

Simenon G. (1963), La colère de Maigret, Paris, Presses de la Cité.

Traduction anglaise: Eglesfield R., San Diego-New York-Londres, Harcourt Brace \& Company, 1980.

- (1951), Maigret et la grande perche, Paris, Presses de la Cité.

Traduction anglaise: Maclaren-Ross J., San Diego-New York-Londres, Harcourt Brace \& Company, 1991.

Thatcher M. (1993), The Downing Street Years, Londres, Harper Collins.

Traduction anglaise: Blot Patricia, Claro Christophe, Denès Hervé, Gnaedig Alain, Mortimer

Philippe et Quadruppain Serge, Paris, Albin Michel, 1993.

Tournier M. (1972), Vendredi ou les limbes du Pacifique, Paris, Gallimard, 1967, «Folio».

Traduction anglaise: Denny N., Harmondsworth, Middlesex, «Penguin Books», 1974. 